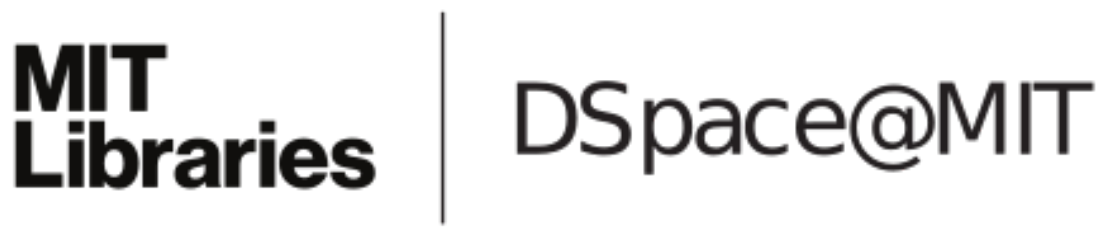

\author{
MIT Open Access Articles \\ Microenvironmental regulation of the \\ sinusoidal endothelial cell phenotype in vitro
}

The MIT Faculty has made this article openly available. Please share how this access benefits you. Your story matters.

Citation: March, Sandra et al. "Microenvironmental Regulation of the Sinusoidal Endothelial Cell Phenotype in Vitro." Hepatology 50.3 (2009): 920-928. Copyright ( 2009 American Association for the Study of Liver Diseases

As Published: http://dx.doi.org/10.1002/hep.23085

Publisher: Wiley Blackwell

Persistent URL: http://hdl.handle.net/1721.1/75328

Version: Author's final manuscript: final author's manuscript post peer review, without publisher's formatting or copy editing

Terms of use: Creative Commons Attribution-Noncommercial-Share Alike 3.0 


\title{
Microenvironmental Regulation of the Sinusoidal Endothelial Cell Phenotype In Vitro
}

\author{
Sandra March ${ }^{1}$, Elliot E. Hui ${ }^{2}$, Gregory H. Underhill ${ }^{1}$, Salman Khetani ${ }^{1}$, and Sangeeta N. \\ Bhatia ${ }^{1,3,{ }^{*}}$ \\ ${ }^{1}$ Harvard-MIT Division of Health Sciences \& Technology, Massachusetts Institute of Technology, \\ Cambridge, Massachusetts \\ ${ }^{2}$ Current address: Department of Biomedical Engineering, University of California, Irvine, CA \\ 92697, USA \\ ${ }^{3}$ Howard Hughes Medical Institute and Electrical Engineering and Computer Science, \\ Massachusetts Institute of Technology/Brigham and Women's Hospital, Boston, Massachusetts
}

\begin{abstract}
Liver Sinusoidal Endothelial Cells (LSEC) differ, both structurally and functionally, from endothelial cells (EC) lining blood vessels of other tissues. For example, in contrast to other EC, LSEC posses fenestrations, have low detectable levels of PECAM-1 expression, and in rat tissue, they distinctively express a cell surface marker recognized by the SE-1 antibody. These unique phenotypic characteristics seen in hepatic tissue are lost over time upon culture in vitro; therefore, this study sought to systematically examine the effects of microenvironmental stimuli, namely, extracellular matrix (ECM) and neighboring cells, on the LSEC phenotype in vitro. In probing the role of the underlying extracellular matrix, we identified collagen I and collagen III as well as mixtures of collagen I/collagen IV/fibronectin as having a positive effect on LSEC survival. Furthermore, using a stable hepatocellular model (hepatocyte-fibroblast) we were able to prolong the expression of both SE-1 and phenotypic functions of LSEC such as Factor VIII activity in cocultured LSECs through the production of short-range paracrine signals. In the course of these experiments, we identified the antigen recognized by SE-1 as CD32b. Collectively, this study has identified several microenvironmental regulators of liver sinusoidal endothelial cells that prolong their phenotypic functions for up to 2 weeks in culture, enabling the development of better in vitro models of liver physiology and disease.
\end{abstract}

\section{Keywords}

endothelial phenotype; SE-1; CD32b; extracellular matrix; hepatocytes

\section{Introduction}

Liver sinusoidal endothelial cells (LSEC) are specialized endothelial cells that play important roles in liver physiology and disease (1). LSEC have several features that distinguish them from other endothelial cells (EC). Morphologically, they are characterized by the presence of open fenestrae arranged in sieve plates, and the lack of an organized basement membrane (2). Functionally, LSEC also can act as scavengers, eliminating soluble waste macromolecules from portal venous blood or immune complexes (3); are critical for

*Corresponding author: Sangeeta Bhatia, MD, PhD., M.I.T., E19-502D; 77 Massachusetts Ave., Cambridge, MA 02139, sbhatia@ mit.edu, Ph: (617) 324-0221. 
inducing CD8+T cell tolerance (4); and synthesize and release Factor VIII, a critical cofactor in the intrinsic coagulation pathway $(5,6)$. Thus, it is evident that a distinct endothelial phenotype is required for the specialized functions of the liver in vivo. Upon isolation from the hepatic microarchitecture, LSEC lose many of their phenotypic functions. We and others have previously demonstrated that the modulation of the hepatocellular phenotypes in vitro can lead to the development of robust in vitro models of some aspects of liver biology $(7,8)$ (29).. Here, we investigate the role of the cellular microenvironment on the LSECs phenotype in vitro with the goal of elucidating fundamental aspects of LSEC biology as well as improving the fidelity of in vitro models of liver tissue.

One of the major challenges in LSEC biology has been the lack of a gold standard biochemical marker for the normal, differentiated LSEC phenotype. The morphological gold standard is the visualization of fenestrations by scanning electron microscopy, a method fraught with technical limitations. The closest biomarker for LSEC phenotype is the antigen recognized by the SE-1 antibody (9). This antibody, unlike those that recognize the ubiquitous endothelial marker CD31, is specific to LSEC in rat tissue, and expression of the SE-1 antigen has been show to positively correlate with the presence of fenestrations.. However, the unknown identity of this antigen has made it difficult to translate findings in the rat liver to other species such as mouse and human or to perturb it with molecular tools such as RNA interference. Over the course of our studies, we sought to identify the antigen for SE- 1 to address these challenges and aid in the mapping of our findings to a medical framework.

When cultured alone after isolation, rat LSEC lose expression of SE-1, increase expression of CD31, and undergo apoptosis after 2 days $(10,11)$. Studies on the role of the microenvironment have shown that perturbations of the underlying matrix can prolong the maintenance of fenestrations (12). Co-cultivation with primary hepatocytes can also prolong the loss of phenotype for several days as measured by continued expression of the SE-1 antigen (13), suppression of CD31 expression (10), and stimulation of proliferation (14). This effect is thought to be due to paracrine signals such as VEGF but limited in duration, in part, due to the concomitant loss of hepatocyte phenotype over several days in culture. In the present study, we exploit two microtechnology platforms previously developed in our group to systematically examine the effect of cell-matrix and cell-cell interactions on LSEC survival and phenotype $(15,16)$. These platforms allow the investigation of LSEC responses to individual matrix species collagen I, collagen III, collagen IV, fibronectin, and laminin and their combinations, as well as enable analysis of the relative impact of contact versus diffusible paracrine signals in co-culture with hepatocytes. In the course of these studies, we also identify the antigen recognized by the SE-1 antibody and map our findings to both mouse and human LSEC.

\section{Materials and Methods}

\section{Isolation and culture of Hepatocytes, Sinusoidal Endothelial cells and fibroblasts}

See supplemental information.

\section{Co-culture of LSEC with neighboring cells}

Cellular co-cultures were performed using a recently developed micromechanical reconfigurable culture method to enable tracking of individual cell types, separation into pure populations for analysis, and deconvolution of contact-mediated versus soluble signals (16). See supplemental information for details. 


\section{Protein Purification, and Identification by Mass Spectrometry}

Isolated LSEC were cultured for one day, and lysed in $1 \mathrm{ml}$ of RIPA buffer (Upstate Biotechnology, Walthem, MA) with protease inhibitors cocktail (Roche, Idianapolis, IN). MAb against SE-1 was covalently coupled to CNBr-activated Sepharose 4B (Amersham, Uppsala, Sweden) and used to affinity-purity the unknown cell surface antigen from LSEC lysates as previously described (17). The isolated protein was identified by peptide mass fingerprinting using LTQ linear ion-trap mass spectrometer (ThermoFisher, San Jose, CA) (supplemental methods).

\section{Rat CD32b Cloning and Transfection}

CD32b cDNA was synthesized (GENEART, Regensburg, Germany) and cloned using the pcDNA3.1 vector. HEK cells were transfected with the rat CD32b or an empty vector using Lipofectamine. Analysis was performed by FACS and Western Blot.

\section{Factor VIII assay}

Factor VIII activity was assayed as previously described using the Coatest C/4 kit (Diapharm, Franklin, OH) (18).

\section{ECM Array}

ECM arrays containing combinatorial mixtures of ECM molecules were fabricated as previously described (15).

\section{Statistical Analysis}

Experiments were repeated 3 times with duplicate or triplicate samples for each condition. For functional assays, one representative outcome is presented. All data are expressed as mean \pm standard deviation. Statistical significance was determined using the Student's $t$ test (Microsoft Excel).

\section{Uptake of acetylated low-density lipoprotein}

Cultured cells were incubated with a medium containing $5 \mu \mathrm{g} / \mathrm{ml}$ of acetylated Low-Density Lipoprotein (LDL)-Alexa 488 conjugated (Molecular Probes, Eugene, OR) for $4 \mathrm{~h}$ at $37^{\circ} \mathrm{C}$ and examined by fluorescence microscopy.

\section{Results}

\section{Expression of endothelial markers in hepatic tissue and in cultured LSEC}

To characterize the phenotype of EC in intact hepatic tissue, and after their isolation and culture, we used several previously described markers for EC present in distinct hepatic vascular beds. These included SE-1, PECAM-1 and RECA. As seen in Figure 1A (top panel), immunohistochemistry with the endothelial marker SE-1 on rat liver sections demonstrated a strong staining of the sinusoidal endothelial cells. No reactivity was observed in central and the portal tract endothelium. In contrast, PECAM-1 was highly expressed in central and the portal tract endothelium and very low expression was detected in the LSEC. The RECA antibody stained the EC of both the sinusoids and large vessels. Next, we assessed the expression of the endothelial markers SE-1, PECAM-1 and RECA, in cultured LSEC at 1 and 3 days after isolation. At day 1, the in vitro expression of SE-1, PECAM-1 and RECA, correlate with the in vivo expression of these markers. Nevertheless, we documented a strong decrease in the expression of SE-1 and an increase in the expression of PECAM-1 at day 3 post-isolation. The expression of RECA did not display significant changes (Figure 1A middle and lower panels and Figure 1B). Scanning electron 
microscopy (SEM) verified that the cell membrane of isolated LSEC at day 1 contained sieve plates with grouped fenestrae (Figure 1A and supplemental Figure 1).

\section{Identification of the antigen recognized by the SE-1 antibody}

To begin linking the expression of the LSEC marker SE-1 to cellular function, we performed affinity purification of the protein recognized by the SE- 1 antibody from LSEC lysates, followed by mass spectrometry analysis These experiments revealed the protein to be CD32b (Figure 2A). To confirm the identity of the recognized protein, LSEC lysates were immunoprecipitated with a SE-1 antibody and the immunoprecipitated material was detected by western blot using two different anti-CD32 antibodies. In both cases the SE-1 antibody and the anti-CD32 recognized the same antigen (Figure 2B). Furthermore, the CD32b cDNA was transiently transfected in HEK cells and probed with the antibody against SE-1. In these experiments, CD32b expressed in HEK cells was recognized by mAb SE-1 using both flow cytometry and western blot analysis (Figure 2C). Inmunohistochemical analyses showed that SE-1 and the polyclonal CD32b antibody staining co-localized in rat liver sections (Figure 2D). We also demonstrated LSEC as the main source of CD32b (supplemental Figure 2). Taken together, these data demonstrate that the SE- 1 antibody recognizes the cell surface receptor CD32b.

The identification of the antigen recognized by SE-1, the CD32b molecule, allowed us to extend our studies to mouse and human LSEC since the SE-1 antibody does not cross-react with these species. We assessed the expression of CD32b in human and mouse LSEC by western blot. In both cases, we showed a downregulation of CD32b over time (Figure 2, E and F), demonstrating that this pattern is conserved across several species. Interestingly, in vivo, expression of CD32b in mouse liver also correlated with patterns of SE-1 staining in rat liver (Supplemental Figure 3C).

\section{Effect of the ECM on the survival of Liver Sinusoidal Endothelial Cells in Culture}

To assess the effects of different components of extracellular matrix on the fate of primary rat LSEC we patterned cells atop combinatorial matrix mixtures of interstitial matrix molecules (fibronectin, collagen I, collagen III) and basement membrane components (laminin, collagen IV), using a microarray platform we have previously described (15). At day 1 after seeding, LSEC expressed high and similar levels of SE-1(CD32b) and low levels of PECAM-1 across all conditions. All islands showed similar number of endothelial cells and absence of apoptotic nuclei on day 1. The presence of attached EC on each island on day 2 and 3 as detected by microscopy was used to score survival over time. A representative part of the array is shown in figure 3A. In contrast to the first day after seeding, notable differences in the number of surviving cells and the percentage of apoptotic nuclei per island emerged over the next 48 hours. The differences in survival and apoptosis were dependent on the initial underlying matrix composition (representative images are shown in figure 3B). Islands containing collagen I or III exhibited the lowest percentage of apoptotic nuclei per island and the highest number of cells per island. In contrast, fibronectin and laminin negatively affected the survival of LSEC, correlating with the highest percentage of apoptotic nuclei per island and the lowest number of cells per island. Factorial analysis revealed that collagen I and collagen III have significant positive effects on cell survival, and laminin has a strong negative effect. We also identified the positive effect of the mixture of collagen I/collagen IV/fibronectin in reducing apoptotic nuclei, and increasing cell number. Interestingly, collagen IV alone did not modulate cell survival; however, in the context of collagen I and III, it had positive effects (Figure 3, C and D). 


\section{Role of supportive cells in maintaining LSEC phenotype}

Our laboratory and others have previously shown that co-culture of hepatocytes with 3T3 fibroblasts can rescue the loss of the hepatocellular phenotype in culture (8), and that both cell contact and short-range paracrine signals were necessary for rescue (16). Here, we used a similar approach to assess the role of supportive cells in maintaining the LSEC phenotype in culture. Co-cultures were performed using a reconfigurable cell culture substrate (16). The system can be configured in 'contact' mode, where contact-mediated signaling can occur between the two populations, or in 'gap' mode, where the populations are separated by an $80-\mu \mathrm{m}$ gap and can only communicate by diffusible soluble signals (Figure $4 \mathrm{~A}$ ). Using this platform, LSEC were cultured together with 3 different combinations of neighboring cells including; a) hepatocytes b) hepatocytes mixed with 3T3 fibroblasts as a 'stable' liver tissue model and c) 3T3 fibroblasts (Figure 4B). In each case, co-cultures were performed in both gap ( $80 \mu \mathrm{m}$ separation) and in contact configuration.

Inmunocytochemical and western blot analysis showed that the expression of SE-1(CD32b) was optimally maintained in cultures combining LSEC with hepatocytes and 3T3 fibroblasts (Figure 4, C and E). We also measured Factor VIII activity as a biochemical marker of LSEC phenotype and Ac-LDL uptake as a measure of the endocytic capability of LSEC. As with SE-1, co-cultures of LSEC with hepatocytes and fibroblasts maintained the highest uptake of Ac-LDL (Figure 4G) and produced the highest levels of Factor VIII. (Figure 4F). No significant Factor VIII activity was detected in hepatocyte or hepatocyte/fibroblast cocultures, confirming that Factor VIII is produced solely by LSEC (data not shown). As expected, albumin secretion- a measure of hepatocellular function-was highest in the cultures containing hepatocyte/fibroblast co-cultures (Figure 4D). Thus, 'tri-cultures' of LSEC with hepatocyte and fibroblasts atop collagen I provided the best cellular microenvironment of the conditions tested for retention of the LSEC phenotype. We also measured LSEC proliferation, while hepatocytes and hepatocytes/fibroblast co-cultures did enhance LSEC proliferation, however, the increase in labeling index of approximately $20 \%$ of nuclei was unlikely to be sufficient to account for the 2- to 3-fold increase in phenotypic functions. In contrast, we did not observe any effect of fibroblasts alone on LSEC (supplemental Figure 4).

We sought to determine whether contact between the cell populations was required for rescue of the LSEC phenotype. Thus, we compared LSECs cultured in gap and contact modes. Western blots of SE-1 (CD32b) demonstrated that the degree of rescue obtained from short-range soluble signals in 'gap' mode was equivalent to that obtained in contact mode (Figure 4H). Thus, our findings indicate that hepatocyte/fibroblast co-culture stabilizes the LSEC phenotype through diffusible factors. In order to identify these soluble factors. First, we examined the expression levels of several cytokines in the conditioned media of the different culture combinations by using a rat cytokine microarray system containing antibodies against 19 cytokines and other proteins. Using this approach, we did not detect any significant changes between this cytokine panel in the well-mixed media after $24 \mathrm{~h}$ of conditioning; however, differences that occur over shorter time scales would not be detectable in this platform (Supplemental Figure 5). Second we performed transcriptome measurements in order to identify soluble factors secreted by hepatocytes upon co-culture with fibroblast. Data from these experiments identified seventeen distinct genes upregulated in hepatocytes and with predicted effects on the endothelium (supplemental Table 1). Thus, in order to assess whether labile diffusible factors could propagate signals between cell populations over longer distance than 80 microns, we performed transwell experiments where LSEC shared their media with hepatocyte/fibroblast co-cultures but were separated by $1 \mathrm{~mm}$. Neither the transwell nor conditioned media models were able to rescue the expression of SE-1(CD32b) as was seen in the short-range 'gap' and contact co-cultures (Figure 4I, and data not shown). 
Finally, we evaluated the longevity of SE-1 expression under different conditions. At day 14, SE-1 (CD32b) expression was only detectable in the 'tri-culture' configuration Notably, over this second week of culture, expression of SE-1 was increased in contact cultures as compared to 80 micron gap culture. Furthermore, in the contact mode at day 14 we observed a migration of the LSEC towards the hepatocyte/fibroblast domain, and these cells express the highest levels of SE-1. These data suggest that reciprocal signaling or contact-mediated signals may play a role over the second week of culture (Figure 4J).

\section{Discussion}

The current study was designed to examine the effect of distinct microenviromental stimuli, in particular extracellular matrix components and paracrine effects of supportive cell types, on the LSEC phenotype. Using two novel platforms, we demonstrated that specific matrix molecules and short-range soluble signals emanating from a relatively stable hepatocellular model (hepatocyte/fibroblast co-cultures) are able to prolong survival and the expression of LSEC phenotypic markers (SE-1 expression and Factor VIII activity) for up to 2 weeks. Notably, the antigen recognized by the antibody SE-1, was identified as CD32b. This particular finding will allow us to better understand the putative role of CD32b (SE-1) on liver sinusoidal endothelial cells, extend this type of study to other species (e.g., mouse and human), and perturb this antigen with molecular tools such as RNA interference.

In our model, the expression of endothelial CD32b was maintained for up to 14 days in an optimal configuration involving co-cultivation of LSEC with both hepatocytes and fibroblasts. CD32b is a low-affinity Fc $\gamma$ receptor constitutively expressed by human adult liver sinusoidal endothelial cells that has the same expression pattern as that previously described for SE-1 in rats, where only the sinusoidal and not the endothelial cells of the large vessels express the antigen (19). Here, we demonstrated that during the process of LSEC differentiation in culture, there is a loss of CD32b expression in rat, mouse and human LSEC, which shares the same dynamics with the loss of the 'gold standard' phenotypic marker, cytoplasmic fenestrations $(20,21)$. CD32b2 plays a major role in the endocytosis of immune complexes in the rat and has been found to be dysregulated in disease, potentially linking the LSEC phenotype with the accumulation of immune complexes observed in some human liver diseases $(19,22)$.

Previous studies have shown that hepatocytes co-cultured with fibroblasts can retain a differentiated hepatocyte phenotype over several weeks in culture $(23,24)$. In this study, we observed that the co-cultivation of LSEC with hepatocyte/fibroblast co-cultures are better able to preserve the hepatocyte phenotype (as determined by the expression of SE-1, up-take of Ac-LDL and Factor VIII activity) than hepatocyte/LSEC cultures under the conditions tested, demonstrating that the 'tri-culture' model has the potential to promote the stabilization of both EC and hepatocytes for up to two weeks. Recent evidence points to the importance of the LSEC as a source of Factor VIII, a critical co-factor in the intrinsic coagulation pathway (6). Our findings on the effect of neighboring cells on LSEC are consistent with observations in the literature. For example, a small population of SE-1 positive cells was observed over 13 days in a 3D perfused co-culture of hepatocytes and LSECs (13) where the hepatocytes in the 3D perfused environment are more phenotypically stable than in monolayer culture (25). In short-term experiments (up to day 4) hepatocytes (that are concurrently losing their phenotype) have been shown to prevent CD31 expression and stimulate proliferation in EC $(10,14)$. Thus, it is reasonable to expect that a monolayer tissue model that combines a relatively stable hepatocyte phenotype (hepatocytes/ fibroblasts) with LSECs would support the LSEC phenotype over a relatively long timescale. Previous studies have shown that LSECs can positively impact hepatocyte function in culture at early time points (24 hours) (26). In comparison, hepatocyte functions in the 3T3 
co-culture model reported here stabilize over approximately 5-7 days. Thus, the generation of 'tri-cultures' to stabilize LSEC function may also have beneficial effects on hepatocyte function early in culture.

Our studies indicate that diffusible factors emanating over short distances, $\mathrm{L}$, $(80$ micron $<\mathrm{L}$ $<1 \mathrm{~mm}$ ), were responsible for the rescue of the endothelial phenotype over the first week in culture. In vivo, it is feasible that hepatocytes and endothelial cells communicate via diffusible signals over short-length scales such as those in the Space of Disse. In an attempt to identify diffusible factors that may play a role in this process, we conducted a comparative analysis of 19 cytokines in the media to determine whether a single factor could be identified to explain these findings; however, no significant differences were identified between supportive (hepatocyte/fibroblast) and non-supportive (fibroblasts or hepatocytes alone) cultures. We also performed a gene expression profiling study comparing hepatocytes and hepatocytes in co-culture with fibroblasts aiming to reveal potential soluble factors that could be contribute to the maintenance of the LSEC phenotype. The candidates revealed by these experiments include pleiotrophin, IGF-I, and angiotensin which potential action in this context correlate with the previously published data (see table 1 , supplementary material)

We also examined the independent role of extracellular matrix coatings on LSEC phenotype. In liver fibrosis, there is a shift in the type of ECM in the space of Disse from the normal low density basement membrane-like matrix (collagen IV, $\mathrm{V}$ and laminin) to interstitial type matrix containing fibril-forming collagen (mainly collagen I and III) (27). In our array, these different matrix and their mixtures were included to simulate the in vivo microenvironment and to capture the transformation of matrix composition observed in liver fibrosis. Our experiments identified the interstitial matrix collagen I and III and mixtures of collagen I/ collagen IV/fibronectin having a positive effect on LSEC survival. These findings are consistent with prior reports that combinations of extracellular matrix can modulate LSEC fenestrations (12); however, the positive role of fibrillar matrix on LSECs in culture was not expected. Alteration of integrin expression or signaling could explain these findings, and in vivo it has been shown that LSEC integrin expression is altered during the process of capillarization in liver cirrhosis (28).

Taken together these studies have revealed several factors that are critical for the maintenance of the functional phenotype of LSEC in culture. This experimental framework should contribute to our understanding of the complex circuitry necessary to maintain cellular function in vivo and in vitro and may provide an in vitro model that captures the phenotype of multiple hepatic cell populations over many days in culture. In the future, incorporation of additional non-parenchymal cells such as cholangiocytes and stellate cells will allow even higher fidelity liver models to be assembled and investigated.

\section{Supplementary Material}

Refer to Web version on PubMed Central for supplementary material.

\section{Abbreviations}

$\begin{array}{ll}\text { LSEC } & \text { Liver sinusoidal endothelial cell } \\ \text { PECAM } & \text { Platelet endothelial cell adhesion molecule } \\ \text { EC } & \text { endothelial cell } \\ \text { RECA } & \text { Rat endothelial cell antigen } \\ \text { Hep } & \text { Hepatocyte }\end{array}$




\section{Acknowledgments}

We thank the Taplin laboratory for his help in mass spectrometry, Steve Katz for the isolations of cells from the rat liver, and Dr. Yaakov Nahmias (MGH) for his help in setting up the LSEC isolation protocol. Financial support was provided by NIH (DK065152 DK56966) and a postdoctoral fellowship to SMR from the Ministerio de Educación y Ciencia y la Asociación Española para el Estudio del Hígado.

\section{References}

1. Aird WC. Phenotypic heterogeneity of the endothelium: II. Representative vascular beds. Circ Res. 2007; 100:174-190. [PubMed: 17272819]

2. Carpenter B, Lin Y, Stoll S, Raffai RL, McCuskey R, Wang R. VEGF is crucial for the hepatic vascular development required for lipoprotein uptake. Development. 2005; 132:3293-3303. [PubMed: 15944181]

3. Smedsrod B, Pertoft H, Gustafson S, Laurent TC. Scavenger functions of the liver endothelial cell. Biochem J. 1990; 266:313-327. [PubMed: 2156492]

4. Limmer A, Ohl J, Kurts C, Ljunggren HG, Reiss Y, Groettrup M, Momburg F, et al. Efficient presentation of exogenous antigen by liver endothelial cells to CD8+ T cells results in antigenspecific T-cell tolerance. Nat Med. 2000; 6:1348-1354. [PubMed: 11100119]

5. Do H, Healey JF, Waller EK, Lollar P. Expression of factor VIII by murine liver sinusoidal endothelial cells. J Biol Chem. 1999; 274:19587-19592. [PubMed: 10391893]

6. Follenzi A, Benten D, Novikoff P, Faulkner L, Raut S, Gupta S. Transplanted endothelial cells repopulate the liver endothelium and correct the phenotype of hemophilia A mice. J Clin Invest. 2008; 118:935-945. [PubMed: 18274668]

7. Allen JW, Bhatia SN. Formation of steady-state oxygen gradients in vitro: application to liver zonation. Biotechnol Bioeng. 2003; 82:253-262. [PubMed: 12599251]

8. Khetani SR, Bhatia SN. Microscale culture of human liver cells for drug development. Nat Biotechnol. 2008; 26:120-126. [PubMed: 18026090]

9. Ohmura T, Enomoto K, Satoh H, Sawada N, Mori M. Establishment of a novel monoclonal antibody, SE-1, which specifically reacts with rat hepatic sinusoidal endothelial cells. J Histochem Cytochem. 1993; 41:1253-1257. [PubMed: 8331290]

10. DeLeve LD, Wang X, Hu L, McCuskey MK, McCuskey RS. Rat liver sinusoidal endothelial cell phenotype is maintained by paracrine and autocrine regulation. Am J Physiol Gastrointest Liver Physiol. 2004; 287:G757-G763. [PubMed: 15191879]

11. Ohi N, Nishikawa Y, Tokairin T, Yamamoto Y, Doi Y, Omori Y, Enomoto K. Maintenance of Bad phosphorylation prevents apoptosis of rat hepatic sinusoidal endothelial cells in vitro and in vivo. Am J Pathol. 2006; 168:1097-1106. [PubMed: 16565486]

12. McGuire RF, Bissell DM, Boyles J, Roll FJ. Role of extracellular matrix in regulating fenestrations of sinusoidal endothelial cells isolated from normal rat liver. Hepatology. 1992; 15:989-997. [PubMed: 1592359]

13. Hwa AJ, Fry RC, Sivaraman A, So PT, Samson LD, Stolz DB, Griffith LG. Rat liver sinusoidal endothelial cells survive without exogenous VEGF in 3D perfused co-cultures with hepatocytes. Faseb J. 2007; 21:2564-2579. [PubMed: 17426068]

14. Krause P, Markus PM, Schwartz P, Unthan-Fechner K, Pestel S, Fandrey J, Probst I. Hepatocytesupported serum-free culture of rat liver sinusoidal endothelial cells. J Hepatol. 2000; 32:718-726. [PubMed: 10845657]

15. Flaim CJ, Chien S, Bhatia SN. An extracellular matrix microarray for probing cellular differentiation. Nat Methods. 2005; 2:119-125. [PubMed: 15782209]

16. Hui EE, Bhatia SN. Micromechanical control of cell-cell interactions. Proc Natl Acad Sci U S A. 2007; 104:5722-5726. [PubMed: 17389399] 
17. March S, Graupera M, Rosa Sarrias M, Lozano F, Pizcueta P, Bosch J, Engel P. Identification and functional characterization of the hepatic stellate cell CD38 cell surface molecule. Am J Pathol. 2007; 170:176-187. [PubMed: 17200192]

18. Rosenberg JB, Foster PA, Kaufman RJ, Vokac EA, Moussalli M, Kroner PA, Montgomery RR. Intracellular trafficking of factor VIII to von Willebrand factor storage granules. J Clin Invest. 1998; 101:613-624. [PubMed: 9449695]

19. Mousavi SA, Sporstol M, Fladeby C, Kjeken R, Barois N, Berg T. Receptor-mediated endocytosis of immune complexes in rat liver sinusoidal endothelial cells is mediated by FcgammaRIIb2. Hepatology. 2007; 46:871-884. [PubMed: 17680646]

20. Shah V, Haddad FG, Garcia-Cardena G, Frangos JA, Mennone A, Groszmann RJ, Sessa WC. Liver sinusoidal endothelial cells are responsible for nitric oxide modulation of resistance in the hepatic sinusoids. J Clin Invest. 1997; 100:2923-2930. [PubMed: 9389760]

21. Tokairin T, Nishikawa Y, Doi Y, Watanabe H, Yoshioka T, Su M, Omori Y, et al. A highly specific isolation of rat sinusoidal endothelial cells by the immunomagnetic bead method using SE-1 monoclonal antibody. J Hepatol. 2002; 36:725-733. [PubMed: 12044521]

22. Muro H, Shirasawa H, Kosugi I, Nakamura S. Defect of Fc receptors and phenotypical changes in sinusoidal endothelial cells in human liver cirrhosis. Am J Pathol. 1993; 143:105-120. [PubMed: 7686339]

23. Kuri-Harcuch W, Mendoza-Figueroa T. Cultivation of adult rat hepatocytes on 3T3 cells: expression of various liver differentiated functions. Differentiation. 1989; 41:148-157. [PubMed: 2612765]

24. Bhatia SN, Balis UJ, Yarmush ML, Toner M. Effect of cell-cell interactions in preservation of cellular phenotype: cocultivation of hepatocytes and nonparenchymal cells. Faseb J. 1999; 13:1883-1900. [PubMed: 10544172]

25. Powers MJ, Janigian DM, Wack KE, Baker CS, Beer Stolz D, Griffith LG. Functional behavior of primary rat liver cells in a three-dimensional perfused microarray bioreactor. Tissue Eng. 2002; 8:499-513. [PubMed: 12167234]

26. Nahmias Y, Casali M, Barbe L, Berthiaume F, Yarmush ML. Liver endothelial cells promote LDL-R expression and the uptake of HCV-like particles in primary rat and human hepatocytes. Hepatology. 2006; 43:257-265. [PubMed: 16440337]

27. Benyon RC, Iredale JP. Is liver fibrosis reversible? Gut. 2000; 46:443-446. [PubMed: 10716665]

28. Couvelard A, Scoazec JY, Feldmann G. Expression of cell-cell and cell-matrix adhesion proteins by sinusoidal endothelial cells in the normal and cirrhotic human liver. Am J Pathol. 1993; 143:738-752. [PubMed: 8362973]

29. Underhill, GH.; Khetani, SR.; Chen, AA.; Bhatia, SN. Liver Tissue Engineering. In: Lanza, R.; Langer, R.; Vacanti, JP., editors. Principles of Tissue Engineering. Third Edition. Elsevier; 2007. 
A

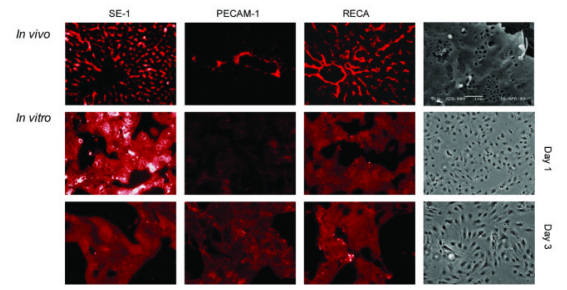

в

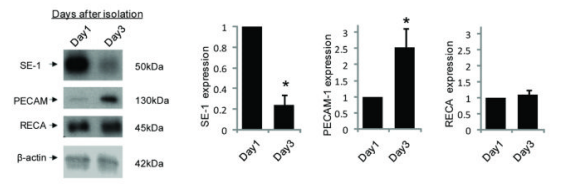

Figure 1. Characterization of LSEC In Vivo and In Vitro

(A) Liver sections of normal rat, or LSEC isolated from rat liver and cultured for 1 or 3 days were stained with the SE-1, anti-PECAM-1 or RECA antibodies. The far right panel shows SEM images of LSEC cultured at day 1 and phase contrast images of LSEC cultured at day 1 or 3 are shown. Fluorescence and phase contrast images were acquired with an original magnification of 20X. (B) Western blotting analyses of total protein from LSEC cultured for 1 or 3 days using the SE-1, anti-PECAM-1 or RECA antibodies. Quantification and normalization are shown in the right panel. $(\mathrm{n}=3+/-\mathrm{SD}, \mathrm{p}<0.05)$ 

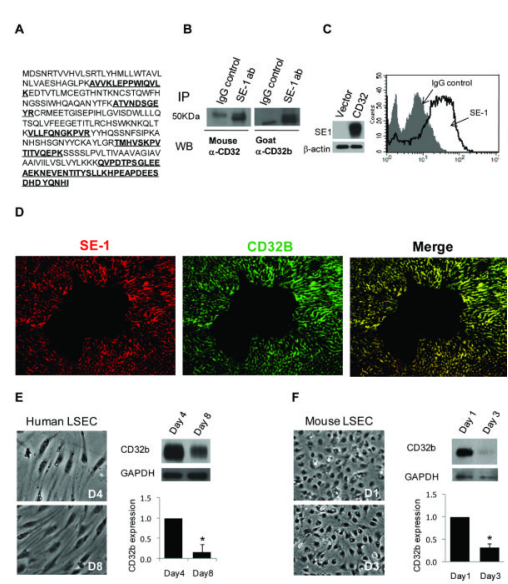

Figure 2. Identification and characterization of the antigen recognized by the SE-1 antibody (A) Amino acid sequences of the rat CD32b molecule identified by mass spectrometry. Bold indicate amino acids predicted by mass spectrometry. (B) LSEC lysate were immunoprecipitated with the SE-1 antibody and the immunoprecipitated material was detected by Western blotting either using the mouse anti CD32 or goat anti CD32b antibodies. (C) HEK cell transfected with rat CD32b cDNA or with the empty vector and analyzed by western blot using the ab SE-1 (right), HEK cells were transfected with the CD32b cDNA and stained with the ab SE-1 or an IgG control and analyzed using FACS. (D) Double immunostaining was performed in liver rat sections using the mAb SE-1 and the goat anti CD32b polyclonal antibody, followed by fluorescently labeled secondary antibodies. Original magnification X20. Total protein lysates of human $(\mathbf{E})$ or mouse $(\mathbf{F})$ LSECs cultured for 4 or 8 days or 1 or 3 days were analyzed by Western blotting using the antibody CD32b 


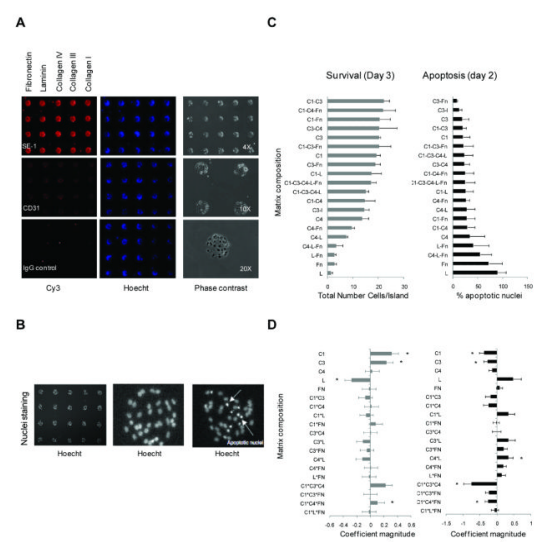

Figure 3. Effect of the ECM components on the survival of cultured LSEC

(A) An example of cells patterned atop an ECM microarray at day 1. Left panel shows staining with the respective antibodies SE-1, anti-PECAM-1 and IgG control following by incubation with $\mathrm{Cy}-3$-conjugated secondary antibody, the corresponding nuclei are visualized by Hoechst staining (central panel), and phase contrast images are seen in the right panel. (B) Representative images of nuclei seen by Hoecht staining with apoptotic nuclei highlighted with arrows. (C) Average and factorial analysis of the percentage of apoptotic nuclei at day 2 observed atop varying matrix compositions. (D) Average and factorial analysis of the total number per cells at day 3 observed atop varying matrix compositions. 
A

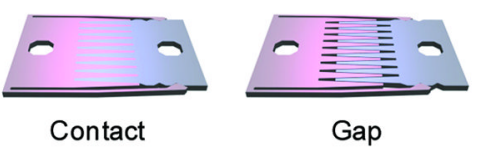

B
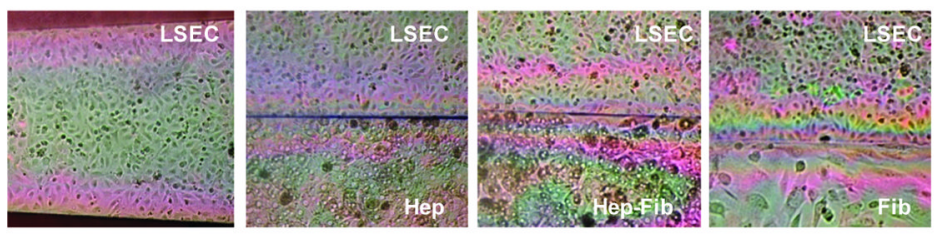

C
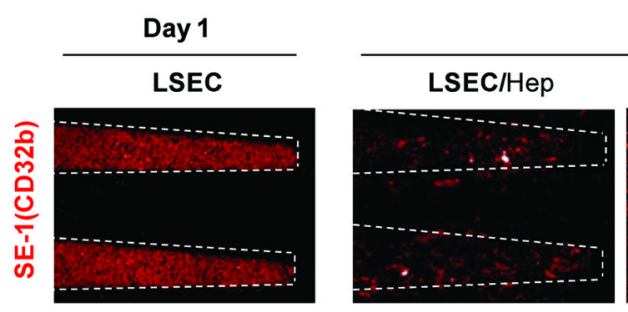

Day 7
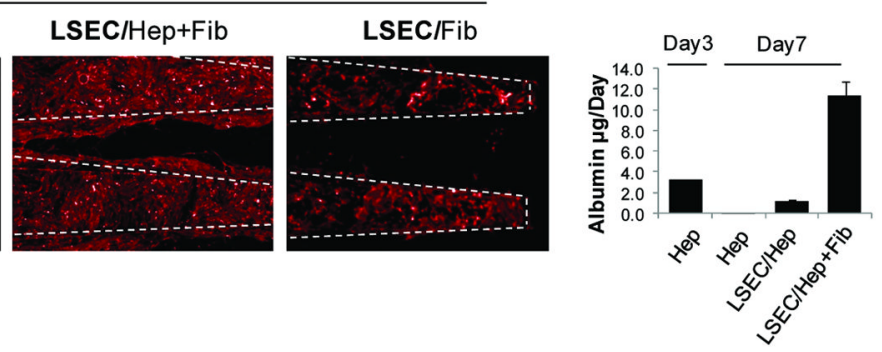

E

F

G
SE-1 (CD32b)

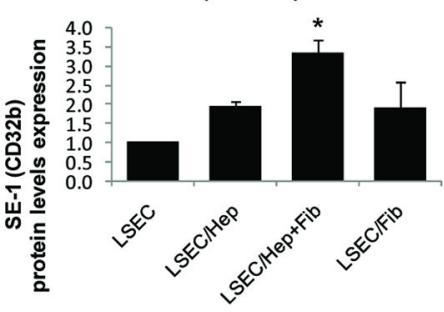

H

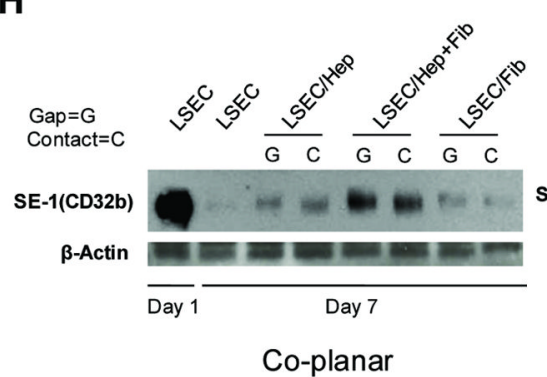

Factor VIII

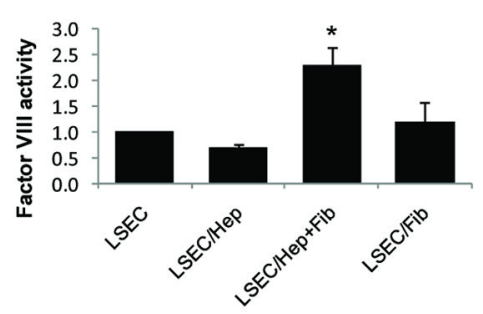

I

J

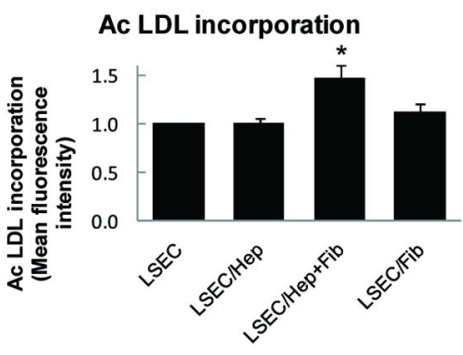

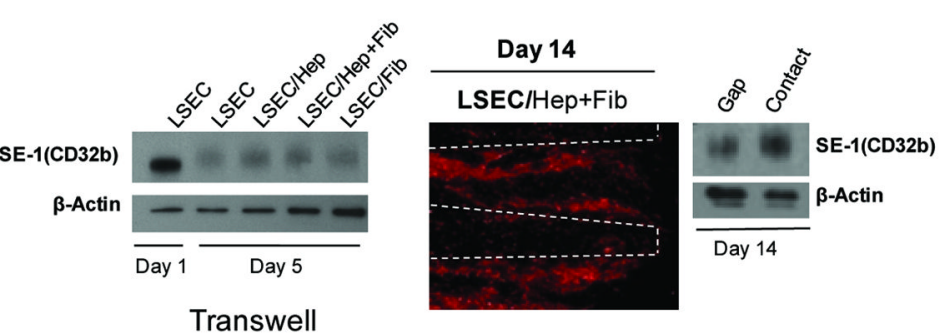

Figure 4. Role of neighboring cells in maintaining LSEC functional phenotype

(A) Schematic representation of the micromechanical platform used for co-culture experiments. (B) Phase contrast images of LSEC co-cultured with 3 different combinations of neighboring cells (C) LSEC co-cultured with 3 differences combinations of neighboring cells were stained with the monoclonal antibody SE-1, followed by conjugated secondary antibody. (D) Albumin secretion by hepatocytes alone or in co-culture with LSEC or LSEC/ fibroblast. (E) SE-1(CD32b) quantification of 3 independent experiments between day 6 and 7. (F) Representative experiment of Factor VIII activity in the supernatants of the different co-cultures.. (G) Uptake of Ac-LDL by the cultured LSEC, a representative experiment of three individual experiments. (H) LSEC co-cultures with 3 different combinations of 
neighboring cells were performed in two modes (gap or contact) for 7 days. Protein lysates of LSECs were analyzed by Western blotting using the antibody SE-1 (CD32b). (I) SE-1 (CD32b) expression on LSEC alone or with 3 different combination of neighboring cells using the transwell system. (J) Inmunostaining and western blot at day 14 using the ab $\mathrm{SE}-1(\mathrm{n}=3,+/-\mathrm{SD} \mathrm{p}<0.05)$ 